\title{
Judging the Abnormalities of Agricultural Machinery by Using Mahalanobis' Distance
}

\section{Choe, Jung Seob}

Department of Precision Mechanical Engineering, Kyungpook National University | Laboratory of Agricultural Machinery and Production Systems Design, Department of Agro-environmental

Sciences, Faculty of Agriculture Kyushu University

\section{Tanaka, Kohe i}

ISEKI Co., Ltd, | Laboratory of Agricultural Machinery and Production Systems Design, Department of Agro-environmental Sciences, Faculty of Agriculture, Kyushu University, Fukuoka

\section{Inoue, $\mathrm{Ei} \mathrm{j} \mathrm{i}$}

Laboratory of Agricultural Machinery and Production Systems Design, Department of Agroenvironmental Sciences, Faculty of Agriculture, Kyushu University

Kim, Tae Wook

Department of Precision Mechanical Engineering, Kyungpook National University | Laboratory of Agricultural Machinery and Production Systems Design, Department of Agro-environmental Sciences, Faculty of Agriculture, Kyushu University

他

https://doi.org/10.5109/1854022

出版情報：九州大学大学院農学研究院紀要. 62 (2)，pp.469-476，2017-09-08. Faculty of Agriculture, Kyushu University

バージョン :

権利関係 : 


\title{
Judging the Abnormalities of Agricultural Machinery by Using Mahalanobis' Distance

\author{
Jung Seob CHOE ${ }^{1}$, Kohei TAKATA ${ }^{2}$, Eiji INOUE, Tae Wook KIMํ, Muneshi MITSUOKA*,
} Takashi OKAYASU and Yasumaru HIRAI
}

\author{
Laboratory of Agricultural Machinery and Production Systems Design, \\ Department of Agro-environmental Sciences, Faculty of Agriculture, \\ Kyushu University, Fukuoka 812-8581, Japan \\ (Received April 28, 2017 and accepted May 10, 2017)
}

\begin{abstract}
This study calculated the Mahalanobis' distance, which is a multidimensional space distance with correlations, from the time-series data of 3-axis translational acceleration and 3-axis rotational angular speed, and examined whether this could be used in judging the abnormalities of the agricultural machineries. As the result, using the Mahalanobis' distance to determine the abnormality of the data was not possible for the changes in not-so-big behaviors, such as turning and temporary stop, but determining the abnormality of the data using the Mahalanobis' distance was clearly possible for sudden changes to the operating status of the equipment, such as a roll over and passing obstacles through an experiment using the model car.

We hypothesized in the beginning that the distribution of the Mahalanobis' distance at the signal space could be separated with the distribution of the Mahalanobis' distance at the unit space. However, unless there is a large-scale change to the behavior, such as a roll over, etc., complete separation is difficult in reality, and we determined realistically that conducting the abnormality determination from the significant difference viewpoint by placing a threshold value to the normalized distribution of the Mahalanobis' distance at the unit space is possible.
\end{abstract}

Key words: abnormality detection, Mahalanobis' distance, time-series data

\section{INTRODUCTION}

Through the development of the processing technology in the semi-conductor recently, variety of small and low-cost sensors have been developed to be applied in diverse machineries, such as mobile communications equipment, including mobile telephone, automobile game equipment, printer, etc. Moreover, collection and storage of the information acquired from sensors, through combination of multiple sensors and data communications devices, have become easy.

In agricultural industry, packaging environment monitoring system, which has integrated multiple sensors and integrated circuit technology (ICT), has been developed, and introduced and used by some largescale farms and agricultural corporations. These systems accumulate packaging environment information, such as temperature, humidity, etc., and work history, and efficient farm work and farming support system are possible based on these data. Moreover, the development of farming robots targeting large-scale packaging is progressing rapidly in Europe and the US, etc., and several small sensors are embedded in the navigation system or the automatic control devices of these robots.

Although development of these advanced farm production technology is progressing rapidly, the 400 farming fatal accidents occurring every year have not been decreasing for decades in Japan, and although it is diffi-

\footnotetext{
1 Department of Precision Mechanical Engineering, Kyungpook National University, Korea

2 ISEKI Co., Ltd, Japan

* Corresponding author (E-mail: mitsuoka@bpes.kyushu-u. ac.jp)
}

cult to find accurate data on similar accidents in Korea, it is speculated that the occurrence rate would not be much different from the Japanese condition. Approximately 70 percent of these farming fatal accidents are fall down/topple from the riding machinery, and over 70 percent of the dead are elderly over 65 years of age, who lacks work agility. As a result, the improving the safety of the agricultural machinery itself to reduce the agricultural machinery accidents, including the farming fatal accidents, is becoming an urgent priority.

In order to resolve the task, first, the establishment of a system to grasp the operating conditions of the agricultural machinery is necessary, and the establishment of the system is expected to become easier through the use of diverse small and low-cost sensors that have been developed, as mentioned earlier (Choe J. S. et al., 2013).

In order to extract characteristics that could evaluate the operating conditions from the information measured by the sensors installed on the equipment, the existing studies conducted the change point detection based on the singular value construction of the vibrating time-series data to determine the vibrating characteristics based on the frequency of each situations and, at the same time, determine the point when the operating conditions change from the vibrating acceleration data of the agricultural machineries (Inoue E., J. Sakai and S. Inaba 1990a,b,c,d, Choe J. S. and E. Inoue 2001). However, because that construction method targets only a single time-series data, when constructing the vibrating time-series from several sensors, the amount of calculation increases dramatically because the singular value analysis must be carried out for each time-series data.

Therefore, this study's final objective is to establish 
an abnormality detection system for the agricultural machineries that could avoid these types of problems and use multiple time-series data. As the first step, we intend to calculate the Mahalanobis' distance (MD), which is a multidimensional (multivariate) space distance from the time-series data of 3-axis translational acceleration and 3-axis rotational angular speed with correlations, and we intend to review first whether we could determine the abnormalities in operational status of the agricultural machineries using this.

\section{THEORETICAL BACKGROUND}

Mahalanobis Taguchi System (MT System) is a method of determining normality and abnormality using the Mahalanobis' distance, which is a multidimensional distance with correlation, and is used in various uses, such as determination of defect in the products during manufacturing process, determination of healthy in health industry, etc. In order to determine abnormality, normal data group and abnormal data group are required. Using the normal data group among multidimensional spaces, the coordinate is determined, and investigates the distribution by calculating the distance between that coordinate and the coordinate of other data. This distance between the coordinates is the Mahalanobis' distance. In this study, we tried to determine the abnormality of the machine's operating status by calculating the Mahalanobis' distance from the 6dimensional vibrating time-series data measured during the operation of the agricultural machineries, and because the theories on the Mahalanobis' distance and MT system are already widely known, we would like to only discuss a few items that are directly related to our study.

\section{Selection of Variables from the SN Ratio}

The SN ratio used in the MT system is a scale that shows the distribution degree of the data. The SN ratio increases as the distribution degree decreases, and the formula changes depending on the target characteristic of the variables. The characteristic of the variable is largely divided into static characteristic and dynamic characteristic. The static characteristic is when the target variable postulates a specific value, and the dynamic characteristic is when the target variable clearly changes by some variable. In other words, the static characteristic shows whether the target fulfills the target value, and the dynamic characteristic focuses on how much the target fulfills the target value.

The purpose of this study is the determination of abnormality of the measured data, so the static characteristic was presumed and the SN ratio of the nominalthe-best characteristic was calculated. The SN ratio in quality engineering is typically used by multiplying 10 times the value presented in common logarithm, as seen in the Formula (1).

In other words, the $\mathrm{SN}$ ratio for the data group from $\mathrm{y}_{1}$ to $\mathrm{y}_{\mathrm{n}}$ is shown as

$$
\mathrm{SN} \text { Ratio }=10 \log _{10}\left(\frac{\mu^{2}}{\sigma^{2}}\right)
$$

and $\mu=\frac{\sum_{i=1}^{\mathrm{n}} \mathrm{y}}{\mathrm{n}}, \sigma=\frac{\sum_{i=1}^{\mathrm{n}}\left(\mathrm{y}_{\mathrm{i}}-\mathrm{y}\right)}{\mathrm{n}-1}$

\section{Orthogonal Table}

The Orthogonal table is an allotment chart with properties that manifests all combinations of that level on the same frequency on a random row, and by using this table, effects of each factor could be independently evaluated without making all possible levels of combination of the given multiple factors.

In this study, the Mahalanobis' distance was postulated as the target variable and used the L12 orthogonal table. Because with the L12 orthogonal table, interaction does not concentrate on one row, and the large bias of the results do not occur often; however, it has a characteristic that the factors in $2^{\text {nd }}$ level cannot be evaluated separately. In this study, in order to increase the accuracy of the abnormal determination, rather than using all 6 data for various data combinations in calculating the Mahalanobis' distance, we looked for the combination with large SN ratio. In the L12 orthogonal table, we established level 1 as "Data Used" and level 2 as "Data Not-Used," and arranged the acceleration and the angular speed in the factor row (Table 1).

Table 1. L12 Orthogonal Table after Arrangement

\begin{tabular}{ccccccc}
\hline & $\alpha_{\mathrm{x}}$ & $\alpha_{\mathrm{y}}$ & $\alpha_{\mathrm{z}}$ & $\omega_{\mathrm{x}}$ & $\omega_{\mathrm{y}}$ & $\omega_{\mathrm{z}}$ \\
\hline 1 & 1 & 1 & 1 & 1 & 1 & 1 \\
2 & 1 & 1 & 1 & 1 & 1 & 2 \\
3 & 1 & 1 & 2 & 2 & 2 & 1 \\
4 & 1 & 2 & 1 & 2 & 2 & 1 \\
5 & 1 & 2 & 2 & 1 & 2 & 2 \\
6 & 1 & 2 & 2 & 2 & 1 & 2 \\
7 & 2 & 1 & 2 & 2 & 1 & 1 \\
8 & 2 & 1 & 2 & 1 & 2 & 2 \\
9 & 2 & 1 & 1 & 2 & 2 & 2 \\
10 & 2 & 2 & 2 & 1 & 1 & 1 \\
11 & 2 & 2 & 1 & 2 & 1 & 2 \\
12 & 2 & 2 & 1 & 1 & 2 & 1 \\
\hline
\end{tabular}

(1=Data Used, $2=$ Data Not-Used)

In order to estimate the ultimate factor combination to be used in the calculation of the Mahalanobis' distance, we calculated the SN ratio of the Mahalanobis' distance in the signaling space from the 6 factor combination using the L12 orthogonal table. The number of combinations when not using the orthogonal table is $2^{6}=64$, and we were able to drastically reduce the number of calculations by using the orthogonal table.

We calculated the SN ratio on the 2 types of intervals, at $2.5 \mathrm{sec}$. and $1 \mathrm{sec}$., of the data from the signal space. This was done to review the appropriate time interval by considering that the data of the signal space changes greatly in a very short time, but the calculation error tend to increase when unnecessary time is 
included

\section{PSD Construction}

Power Spectral Density (PSD) is showing the frequency information on each frequency band of the physical quantity included in certain signals. By observing the PSD size for each frequency by PSD construction of the signal data, the characteristic of that signal could be determined.

In this study, PSD was used as an index of the section length of the data used in the Mahalanobis' distance calculation and $\mathrm{SN}$ ratio calculation. When using the Mahalanobis' distance in abnormality determination, it is speculated that the data range of the frequency that becomes the characteristic of that signal, meaning the PSD's peak is very high, must be sectioned longer. This is because the data from the high peaked frequency range has big impact in that data group, and the peak deriving from the movement from both the normal and the abnormal data needs to be included in the Mahalanobis' distance calculation.

\section{MATERIALS AND METHOD}

\section{MEMS Sensor}

In order to obtain vibrating time-series data used in calculating the Mahalanobis' distance, translational 3axis acceleration and rotating 3-axis angular speed of a combine during the cutting activity were measured. Previously, a gyro sensor was generally used to measure the angular speed of a moving object, but due to the cost and the large size, its use was limited to large moving objects. However, due to a rapid development of the semi-conductor technology recently, small and low cost

Table 2. Specification of an MEMS Sensor, MVP-SDA3-BC

\begin{tabular}{ll}
\hline Measuring Range & $\pm 20 / \pm 60\left[\mathrm{~m} / \mathrm{s}^{2}\right]$ \\
Sample Period & $0.5,1,2,5,10,50,100,200,500[\mathrm{~ms}]$ \\
Memory Time & $\begin{array}{l}33 \text { [hours] } \\
\text { (1 GB SD Card, during } 0.5 \text { msec sampling) }\end{array}$ \\
Power & $1700[\mathrm{mAh}]$ (Lithium-ion secondary cell) \\
Battery Operating & 50 [hours] \\
Time & (During 5 ms continuous movement sam- \\
& pling) \\
Body Size & $50[\mathrm{~mm}] \times 75[\mathrm{~mm}] \times 20[\mathrm{~mm}]$ \\
Weight & $120[\mathrm{gf}]$ \\
\hline
\end{tabular}

Table 3. Specification of an MEM Sensor, 3DM-GX3-45

\begin{tabular}{ll}
\hline Azimuth Range & 3 -axis each $360[\mathrm{deg}]$ \\
Acceleration Range & $49\left[\mathrm{~m} / \mathrm{s}^{2}\right]$ \\
Angular Speed Range & $300[\mathrm{deg} / \mathrm{s}]$ \\
Supplied Voltage & Maximum $9[\mathrm{~V}]$ \\
Supplied Current & $0.08[\mathrm{~A}]$ \\
Body Size & $44[\mathrm{~mm}] \times 24[\mathrm{~mm}] \times 14[\mathrm{~mm}]$ \\
Weight & $23[\mathrm{~g}]$
\end{tabular}

sensors, called MEMS, have been developed and are being used. In this study, MVP-SDA3-BC MEMS sensor, a product from Microstone, with the specifications set forth in Table 2, was selected to measure the acceleration and angular speed of a combine. For measuring the acceleration and angular speed of a model car, even smaller sensor, 3DM-GX3-45, a product from Microstrain, shown in Table 3, was used.

\section{Cutting Work Experiment}

The testing equipment used in this rice harvesting experiment was a heading-feeding combine, manufactured by M, model VM15G, as seen in Table 4 . At the rice packing of a farm affiliated with Kyushu University, the MVP-SDA3-BC sensor was attached to the upper part of the frame of the left-front side of the machine. The translational 3-axis acceleration and rotating 3-axis angular speed of the machine was measured at the sampling frequency of $200 \mathrm{~Hz}$ for each of the operating status, idling status, cutting work status, cutting, threshing, etc. 3-axis acceleration and 3-axis angular speed are each recorded as $\alpha_{\mathrm{x}}, \alpha_{\mathrm{y}}, \alpha_{\mathrm{z}}, \omega_{\mathrm{x}}, \omega_{\mathrm{y}}$, and $\omega_{\mathrm{z}}$.

Table 4. Data of the Testing Combine

\begin{tabular}{llc}
\hline Body Size & Full Length (mm) & 3210 \\
& Full Width (mm) & 1670 \\
& Full Height (mm) & 1980 \\
& Body Weight (kgf) & 1320 \\
\hline $\begin{array}{ll}\text { Threshing and Sorting } \\
\text { Part }\end{array}$ & $\begin{array}{l}\text { Threshing Cylinder Speed of } \\
\text { Revolution (rpm) }\end{array}$ & 470 \\
& $\begin{array}{l}\text { Discharge Cylinder Speed of } \\
\text { Revolution (rpm) }\end{array}$ & 1410 \\
\hline
\end{tabular}

\section{Drive Test of Model Car}

Because conducting an experiment with an actual combine regarding situations such as engine failure or a fall is difficult, a small model car was produced for a comparative experiment. The model car shown in the Diagram 1 is a $2-$ wheel drive by a motor, and the specifications are length of $140 \mathrm{~mm}$, width of $94 \mathrm{~mm}$, center height of $45 \mathrm{~mm}$, weight of $740 \mathrm{gf}$ and tire diameter of $32 \mathrm{~mm}$. 3-axis translational acceleration and 3-axis angular speed were each measured for driving on hard

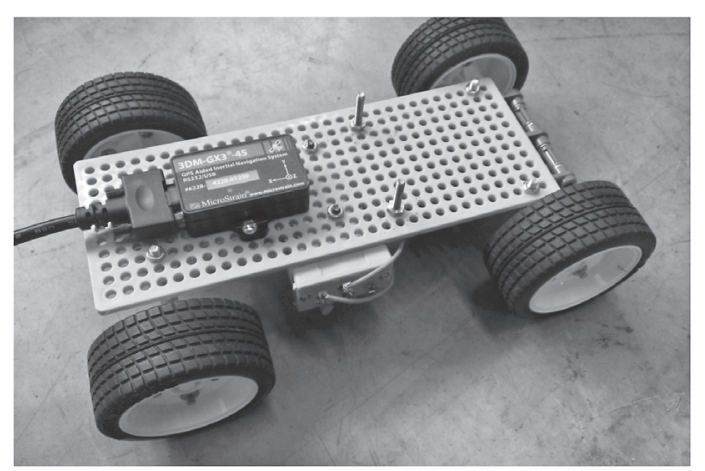

Diagram 1. Outside of the Model Car. 
flat surface, slope, and crossing obstacle and a fall as a result. The angle of inclination of the slope was 25 degrees, as seen in the Diagram 2, and the height of the obstacle was $8 \mathrm{~mm}$, with an angle of inclination at 15 degrees.
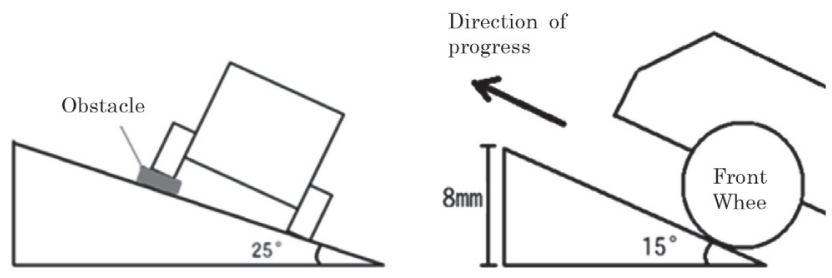

Diagram 2. Crossing of an Obstacle by the Model Car (Front View, Side View).

\section{RESULTS AND DISCUSSION}

\section{PSD Construction}

In order to reveal the characteristics of the frequency of time-series data of the acceleration and the angular speed obtained from the combine work experiment, the PSD was calculated on 3,000 data unit spaces (15 sec section). The Diagram 3 shows the PSD on the translational acceleration in the $\mathrm{x}$-axis direction (progress direction), and the Diagram 4 shows the PSD on the angular speed of the y-axis circumference.

From the fact that the first vibration of an engine rotating at $3,000 \mathrm{rpm}$ is $50 \mathrm{~Hz}$ and the first vibration of the threshing cylinder in the threshing and sorting part rotating at $470 \mathrm{rpm}$ is approximately $8 \mathrm{~Hz}$, it is easily understood that the peak appearing from this section derives from these. In other results, the size of the peak is different, but the location of the occurrences showed

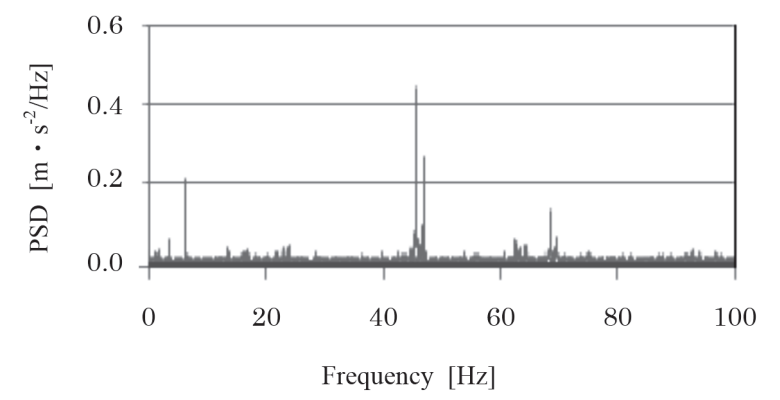

Diagram 3. PSD of $\alpha_{\mathrm{x}}$

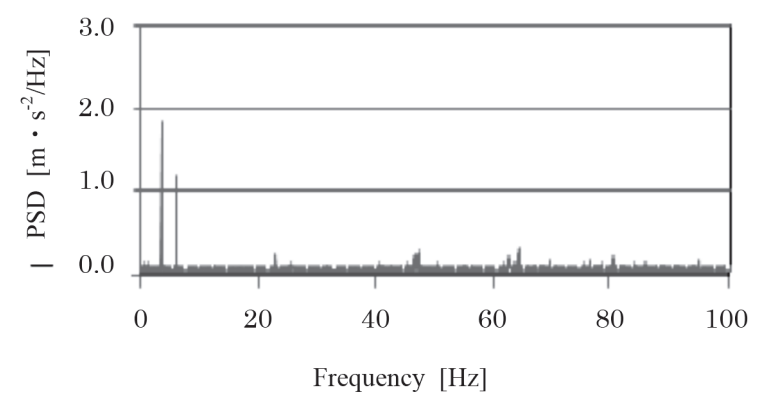

Diagram 4. PSD of $\omega_{y}$. almost identical appearances as the two diagrams. Considering that a peak appeared around $70 \mathrm{~Hz}$, although not large, it was determined that the signal space would be better to use the time-series data up to approximately $100 \mathrm{~Hz}$ section.

\section{Calculation of the SN Ratio}

SN ratio was calculated for the Mahalanobis' distance of each of the combinations of the 6 variables using the L12 orthogonal table. As with the PSD construction, SN ratio was calculated for signal space of 500 data (2.5 sec. duration) and 200 data (1 sec. duration), and compared the results. Sampling was done twice in the section under the same conditions, and the SN ratio was calculated for each.

The Diagrams 5 and 6 shows the SN ratio at turning and sudden stop at $2.5 \mathrm{sec}$. space. At turning, the values are almost identical, except for the combinations 9 and 11, but at sudden stop, special regularities cannot be found in the relationship between the combination of the variables and the size of the $\mathrm{SN}$ ratio. It is speculated that the reason for a larger distribution of the SN ratio from the combination of variables during a sudden stop, as compared to turning, is due to the fact that the car's condition changes are much bigger during a sudden stop.

The Diagrams 7 and 8 show the SN ratio at turning and sudden stop at 1 sec. space each. Here, the SN ratio size showed somewhat similar tendencies according to

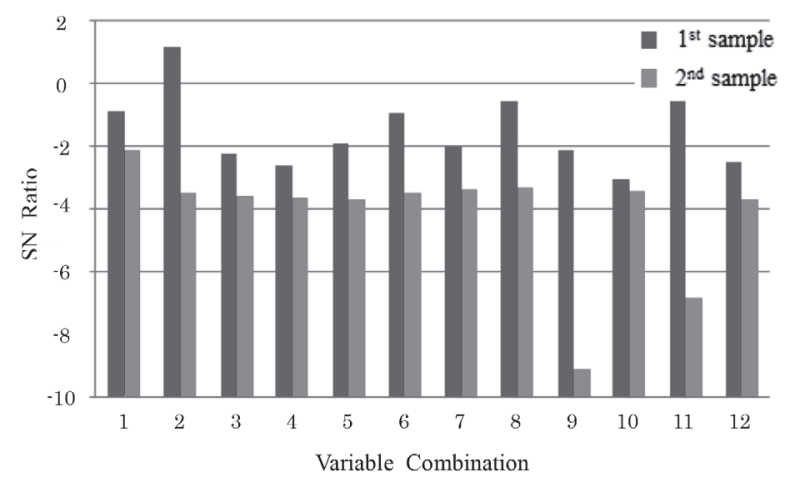

Diagram 5. Data's SN Ratio at Turning (2.5 sec. duration).

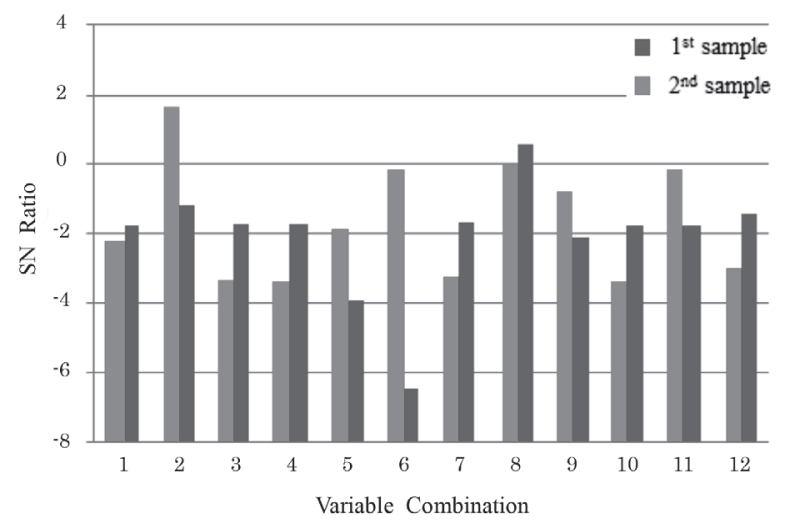

Diagram 6. Data's SN Ratio at Sudden Stop (2.5 sec. duration). 


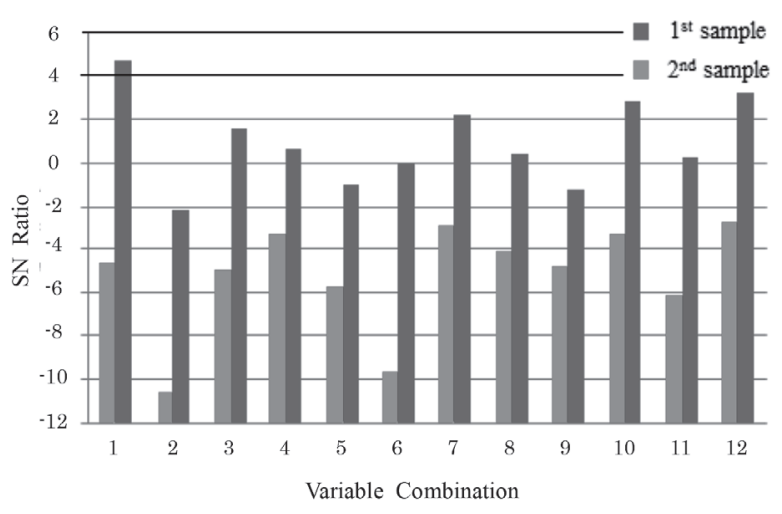

Diagram 7. Data's SN Ratio at Turning (1 sec. duration).

the combination of variables for the combine's each

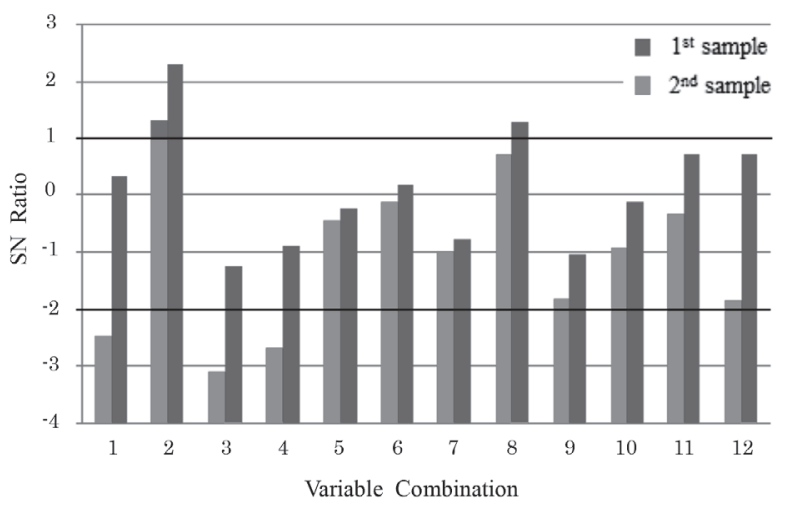

Diagram 8. Data's SN Ratio at Sudden Stop (1 sec. duration).

behavior. It seems that this is due to the fact that as the sampling section narrowed, the amount of noise included has lessened, and the impact on the changes to the data due to the combine's behavior has comparatively increased.

The combination of variables with large SN ratio is different according to the combine's behavior, and this is due to the fact that the combine's behavior has a large impact on certain variables. As such, it is natural for the $\mathrm{SN}$ ratio to be large for a combination with that variable. For example, because the SN ratio for the combination 1 (using all 6 variables) is comparatively larger than other

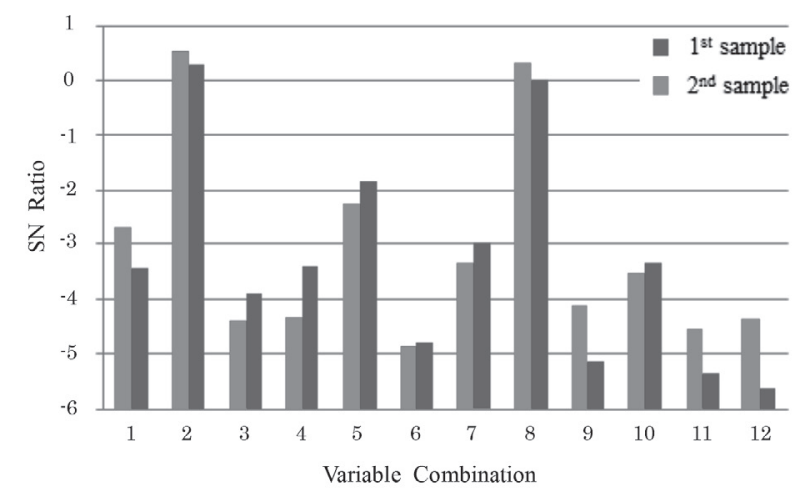

Diagram 9. SN Ratio from the Data During Sideway Overturning of a Model Car (1 sec. duration). combinations at the combine's 2 types of behaviors, when comparing data measured from other behavior, it is appropriate to use all 6 data of the 3 -axis translational acceleration and 3-axis angular speed.

As shown in the Diagram 9, when the SN ratio was calculated from the data during the sideway overturning at a rigid slope by a model car using the same method, the tendency was similar to that of an actual combine during a sudden stop. In other words, the change of the status from passing an obstacle or rollover of a car means a switch to a sudden change in speed (acceleration) from a near inertial status of forward driving, similar to a sudden stop.

\section{Mahalanobis' Distance}

The Mahalanobis' distance was calculated at 3,000 data ( 15 sec. duration), as with the PSD construction, for the unit space, and 500 data (2.5 sec. duration) and 200 data ( $1 \mathrm{sec}$. duration) for the signal space. The operational status of the combine and the model car at unit space and signal space is shown in Table 5.

Table 5. Data Allocation for the Calculation of the Mahalanobis' Distance

\begin{tabular}{lll}
\hline Testing Machine & Unit Space & $\begin{array}{l}\text { Forward (Threshing, Cutting } \\
\text { Non-Operating) }\end{array}$ \\
& Signal Space & $\begin{array}{l}\text { Turning } \\
\text { Sudden Stop }\end{array}$ \\
\hline Model Car & Unit Space & Forward at Rigid Flat Surface \\
& Signal Space & Forward at Rigid Slope \\
& Passing Obstacle at Rigid Slope \\
& Sideway Overturning at Rigid \\
& Slope \\
&
\end{tabular}

The Mahalanobis' distance at signal space is calculated based on the normalized data of unit space. In this study, in order to evaluate the distribution of the Mahalanobis' distance at the unit space and the signal space nearing the normalized distribution curve and the legitimacy of the determination of the variables, we calculated the distribution of the Mahalanobis' distance at the unit space of the same combination. Because the regular distribution coefficient is on the vertical-axis of the graph and the Mahalanobis' distance is on the horizontal-axis, it means that there are more data indicating the value of the Mahalanobis' distance exists in that signal space as the coefficient is larger.

Because the $\mathrm{SN}$ ratio is larger as the distribution is less, as shown in the Table 6, the Mahalanobis' distance was calculated for the combination of 7 and 11 at turn-

Table 6. Combination of 6 Variables with Large SN Ratio

\begin{tabular}{lll}
\hline During Turning & 7 & $\alpha_{y}, \omega_{y}, \omega_{z}$ \\
& 11 & $\alpha_{z}, \omega_{y}$ \\
During Sudden Stop & 2 & $\alpha_{x}, \alpha_{y}, \alpha_{z}, \omega_{x}, \omega$ \\
& 8 & $\alpha_{y}, \omega_{x}$ \\
\hline
\end{tabular}


ing, combination of 2 and 8 at sudden stop, which has a relatively large $\mathrm{SN}$ ratio compared to the other combinations, and this was compared to the Mahalanobis' distance of combination 1, which used all 6 variables.

Signal Space Using the Measured Data from the Testing Equipment

The Diagrams 10 and 11 show the distribution of the Mahalanobis' distance at turning and at sudden stop, each.

In all situations, the Mahalanobis' distance using the combination 1, which used all 6 variables, showed wider and lower normal distribution probability density function curve as compared to the other two combinations. This means that when using all 6 variables, the freedom increases and includes more error, and a more distributed data is acquired.

When comparing the $2.4 \mathrm{sec}$. duration graph and the 1 sec. duration graph in each of the signal spaces, the distribution of the Mahalanobis' distance of the combination 1 do not show much change, but the distribution of the Mahalanobis' distance of the combination with large $\mathrm{SN}$ ratio show changes. However, the normal distribution probability density function did not show the similar changes for the number of data at the signal space, and this was due to the fact that the normal distribution probability density function is greatly impacted by the characteristics of the variables within the sampling section, as well as the degree of freedom of data.

When looked at closely, for the combination 7 from the Diagram 10 and the combination 2 from the Diagram 11 , it is noticed that the distribution lessens and the mean increases as the sampling time is shortened. It could be speculated that the characteristic of distribution of data by the movement of the testing equipment is more clearly reflected because the noise within the signal space decreases as the sampling time interval of the data becomes smaller.
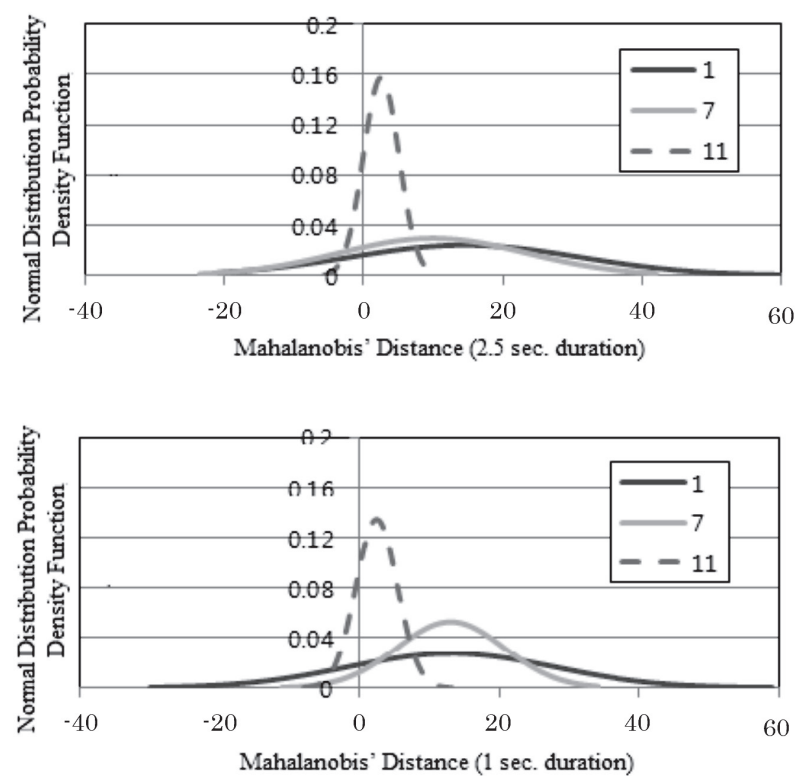

Diagram 10. Distribution of the Mahalanobis' Distance at Turning.
On the other hand, for the combination 11 from the Diagram 10 and the combination 8 from the Diagram 11, the shape of the function curve does not change much even when the length of the signal space changes, and this may be due to the fact that the number of variables used for the two combinations is very small, at 2 , and these variables are not much impacted by noise naturally.
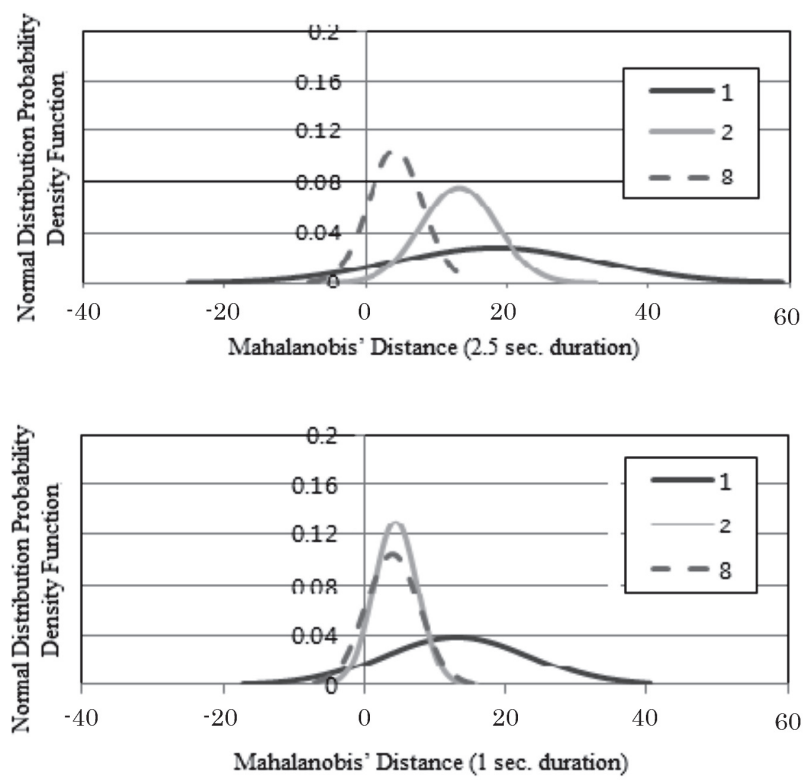

Diagram 11. Distribution of the Mahalanobis' Distance at Sudden Stop.

Comparison with the Unit Space and the Signal Space Using the Measured Data from the Testing Equipment

The Mahalanobis' distance was calculated for the unit space with the same combination of variables as with the signal space. As the result, only the combination 2 had a larger normal distribution probability density function than when using all 6 variables, as can be seen in the Diagram 12. Overall, data tended to distribute wider as the number of variables used for calculation gets smaller. Because the Mahalanobis' distance at the signal space is achieved based on the standardized unit space, it may not be sufficient to select variables with only the $\mathrm{SN}$ ratio calculated at the signal space.

When comparing the distribution of the

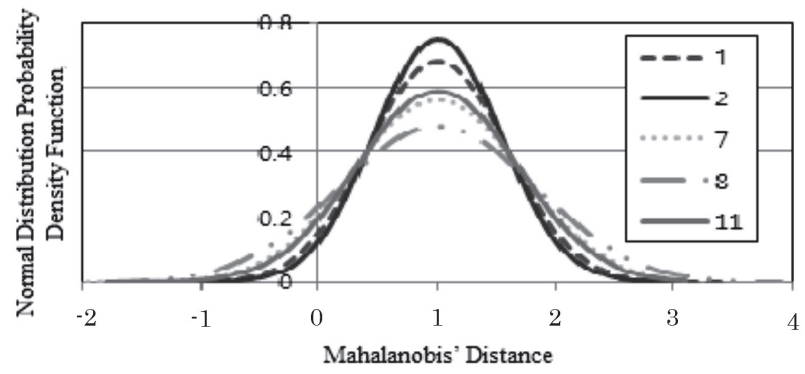

Diagram 12. Distribution of the Mahalanobis' Distance of Unit Space. 
Mahalanobis' distance at the signal space and the unit space with 2 combinations of large SN ratio, as seen in the Diagram 13, there were parts where the signal space and the unit space overlap for both turning and sudden stop, but because the distribution curve of the unit space leaned towards the left limit, it was determined that the SN ratio could be used as one of the determination index for abnormality.
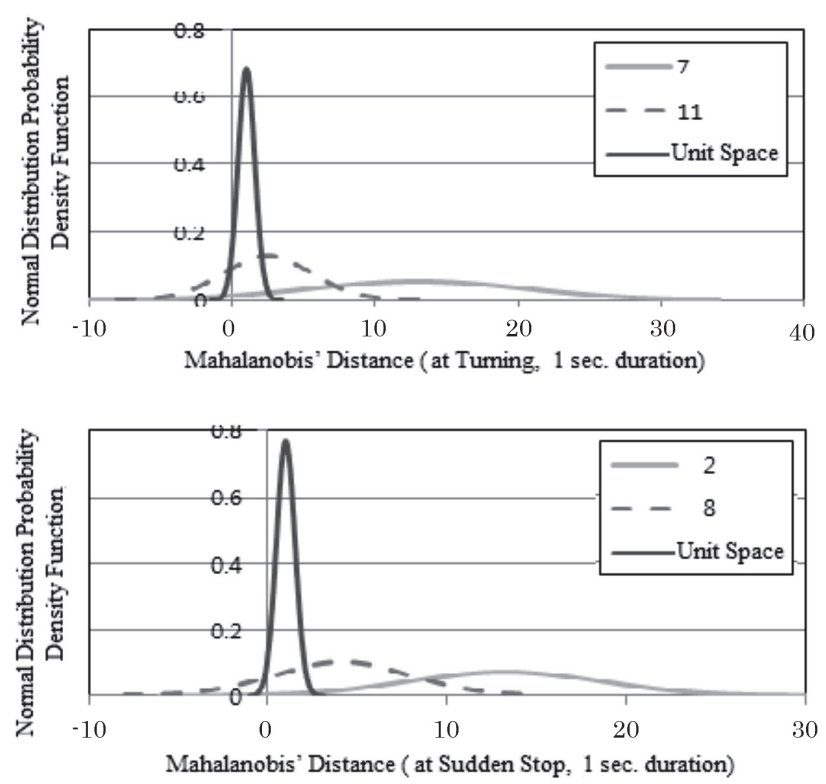

Diagram 13. Comparison of the Mahalanobis' Distance at Signal Space and Unit Space.

The Mahalanobis' Distance of the Measured Data from the Model Car

Measuring data under clearly abnormal circumstances, such as engine failure or roll over, is very difficult to carry out with the actual combine, so a model car was produced and the testing was carried out.

The Diagram 14 shows the Mahalanobis' distance distribution curve for roll over, passing obstacle, slope driving, the unit space (flat surface driving) of a model car. The Mahalanobis' distance for all cases were calculated using all 6 variables of 3-axis translational acceleration and 3-axis rotational angular speed.

As shown in the Diagram 14, unlike the results using the testing equipment, the distribution of the

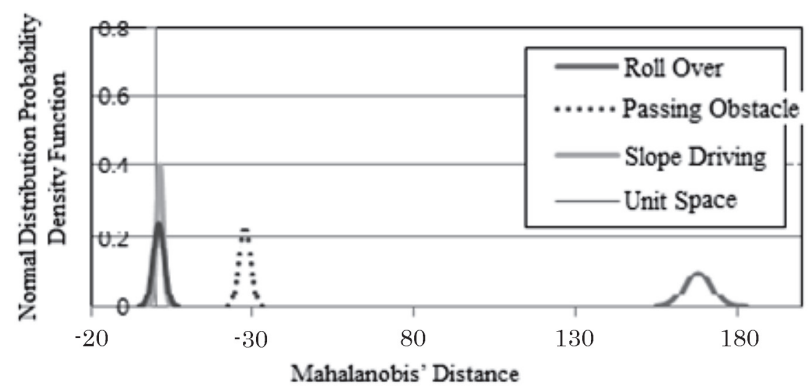

Diagram 14. The Mahalanobis' Distance at Various Operating Status of the Model Car.
Mahalanobis' distance at each status showed clear differences in a test using the model car. The distribution curve during the slope driving almost overlaps with the distribution curve of the unit space, and can be determined as a normal condition. The distribution of the Mahalanobis' distance at passing obstacles and in roll over state are completely separated from the unit space, and these could be determined as data with high abnormalities. This signifies that the Mahalanobis' distance could be very useful in judging abnormalities of the combine, other agricultural machineries and cars.

\section{CONCLUSION}

This study calculated the Mahalanobis' distance, which is a multidimensional space distance with correlations, from the time-series data of 3-axis translational acceleration and 3-axis rotational angular speed, and examined whether this could be used in judging abnormalities of the agricultural machineries. In order to increase the accuracy of the abnormality determination using the Mahalanobis' distance, the PSD was used as an index for deciding the section width of the data used in calculating the Mahalanobis' distance and the computation of the SN ratio, and the SN ratio was used in selecting the factors used for the computation of the Mahalanobis' distance.

As the result, using the Mahalanobis' distance to determine the abnormality of the data was not possible for the changes in not-so-big behaviors, such as turning and temporary stop, but determining the abnormality of the data using the Mahalanobis' distance was clearly possible for sudden changes to the operating status of the equipment, such as roll over and passing obstacles through an experiment using the model car.

The combination of factors with large SN ratio had increased usefulness as a data because the deviation of the Mahalanobis' distance calculated from it gets smaller; however, even when the SN ratio gets larger, if the number of factors used in the calculation decreases, the median value of the distribution of the Mahalanobis' distance gets closer to 1 and overlaps with the unit space. This means that when the behavior of the combine changes, this not only impacts a certain specific factor, but causes chaos to data of almost all factors. Therefore, when calculating with a small number of factors, the abnormality of the data also gets smaller, so it causes the problem of making the determination of abnormality inaccurate. Moreover, the combination of the factors that increases the $\mathrm{SN}$ ratio changes as the combine's behavior changes, and this is estimated due to the fact that the acceleration and the angular speed that have increasing impact changes according to the behavior changes. Therefore, when selecting the factors used for the calculation of the Mahalanobis' distance by simply the size of the SN ratio, the median value of the distribution of the Mahalanobis' distance must also be considered, and in conclusion, this method was determined to be not very useful.

We hypothesized in the beginning that the distribu- 
tion of the Mahalanobis' distance at the signal space could be separated with the distribution of the Mahalanobis' distance at the unit space. However, unless there is a large-scale change to the behavior, such as a roll over, etc., complete separation is difficult to achieve in reality, and we determined realistically that conducting the abnormality determination from the significant difference viewpoint by placing a threshold value to the normalized distribution of the Mahalanobis' distance at the unit space is possible.

\section{AUTHOR CONTRIBUTIONS}

J. Choe designed the study, analyzed the data and wrote the paper. K. Takata performed the scale model experiments. T. Okayasu and Y. Hirai participated in the design of the study and performed the experiments. E. Inoue and M. Mitsuoka designed the study, supervised the work, wrote the paper and provided facilities and resources. W. Kim performed the statistical analysis. All authors assisted in editing of the manuscript and approved the final version.

\section{ACKNOWLEDGEMENTS}

This Research was supported by Kyungpook National University Research Fund, 2015-2017.

\section{REFERENCES}

Choe J. S. and E. Inoue 2001 A Study on the Vibration Characteristics of a Head-Feeding Combine by Spectral Analysis. Journal of the Korean Society for Agricultural Machinery, 26(1): 11-20

Choe J. S., M. Mitusoka, E. Inoue, T. Okayasu, Y. Hirai, T. W. Kim, S. G. Kwon and J. M. Park 2013 Measuring Inclination Angle of Semi-crawler Tractors Using MEMS Sensor. Journal of the. Faculty of Agriculture Kyushu University, 58: 79-85

Inoue E., J. Sakai and S. Inaba 1990a Basic Studies on Vibration Characteristics of the Rubber Crawler System for Farm Machinery (Part 1), Journal of the Japanese Society of Agricultural Machinery, 52(1): 27-34

Inoue E., J. Sakai and S. Inaba 1990b Basic Studies on Vibration Characteristics of the Rubber Crawler System for Farm Machinery (Part 2), Journal of the Japanese Society of Agricultural Machinery, 52(4): 29-36

Inoue E., J. Sakai and S. Inaba 1990c Basic Studies on Vibration Characteristics of the Rubber Crawler System for Farm Machinery (Part 3), Journal of the Japanese Society of Agricultural Machinery, 52(5): 11-18

Inoue E., J. Sakai and S. Inaba 1990d Basic Studies on Vibration Characteristics of the Rubber Crawler System for Farm Machinery (Part 4), Journal of the Japanese Society of Agricultural Machinery, 52(6): 19-26 